\title{
On the energy-momentum tensor of light in strong fields: an all optical view of the Abraham-Minkowski controversy
}

\author{
Alexander J. Macleod, Adam Noble, and Dino A. Jaroszynski \\ Department of Physics, SUPA \& University of Strathclyde, Glasgow G4 0NG, United Kingdom
}

\begin{abstract}
The Abraham-Minkowski controversy is the debate surrounding the "correct" form of the energy-momentum tensor of light in a medium. Over a century of theoretical and experimental studies have consistently produced conflicting results, with no consensus being found on how best to describe the influence of a material on the propagation of light. It has been argued that the total energy-momentum tensor for each of the theories, which includes both wave and material components, are equal. The difficulty in separating the full energy-momentum tensor is generally attributed to the fact that one cannot obtain the energy-momentum tensor of the medium for real materials. Non-linear electrodynamics provides an opportunity to approach the debate from an all optical set up, where the role of the medium is replaced by the vacuum under the influence of a strong background field. We derive, from first principles, the general form of the energy-momentum tensor in such theories, and use our results to shed some light on this long standing issue.
\end{abstract}

Keywords: Abraham-Minkowski controversy, slow light experiments, non-linear vacuum electrodynamics, EulerHeisenberg, Born-Infeld

\section{INTRODUCTION}

The momentum of light in the linear vacuum is an unambiguous concept, with decades of theoretical and experimental confirmation. However, at the beginning of the 20th century a debate concerning the momentum of light in a medium emerged within the physics community which is still a source of controversy to this day. Often referred to as the "Abraham-Minkowski controversy", it has its origins in the two rival expressions obtained by Minkowski ${ }^{1,2}$ and Abraham ${ }^{3,4}$ for the momentum of light within a medium, which differ by a factor of $n^{2}$, where $n$ is the refractive index of the medium. The discrepancy between these two forms, which both have a sound theoretical basis, ${ }^{5}$ led to numerous investigations through the latter half of the century which attempted to pin down the "correct" form.

This led to experimental and theoretical work supporting Minkowski ${ }^{6-10}$ and Abraham, ${ }^{11-15}$ and it is this conflicting evidence which has been the source of much of the confusion surrounding this subject. What many of these studies did not fully take into account was the importance of the energy-momentum tensor of the material itself. When the response of the medium to the presence of the electromagnetic field is taken into account consistently, it has been argued that Abraham and Minkowski are then equivalent. ${ }^{16,17}$ More recently, the debate has been claimed as resolved, ${ }^{18}$ with both forms of the momentum being physically relevant, such that the Abraham form is attributed to the kinetic momentum, and the Minkowski form to the canonical momentum.

It has been argued (see for example ${ }^{19}$ ) that various studies which found particular forms of the momentum of light (for example ${ }^{20-22}$ ) incorrectly modelled the medium through which the light was propagating. Herein lies the major issue being faced by research into the Abraham-Minkowski controversy; calculating the material component of the energy-momentum tensor is non-trivial even for idealised media. So for real materials it is often not possible to accurately calculate the material component, which has obvious implications for analysing any experiment which could be performed to potentially discriminate between, or show the equivalence of, Abraham and Minkowski. With this in mind, it is reasonable to ask whether there is another way of looking at this

Further author information: (Send correspondence to A.J.M.)

A.J.M.: E-mail: alexander.macleod@strath.ac.uk

A.N.: E-mail: adam.noble@strath.ac.uk 
issue which avoids the problems associated with modelling the medium, and could be more readily adapted to experiment.

An interesting prospect is offered by turning to non-linear vacuum electrodynamics. This is the umbrella term given to generalisations of classical electromagnetism which allow for real photon-photon interactions. The two most prominent non-linear theories are those due to Euler and Heisenberg, ${ }^{23-25}$ and Born and Infeld. ${ }^{26,27}$ Euler-Heisenberg theory is obtained through integrating out the fermionic degrees of freedom in the path integral of quantum electrodynamics (QED), which then allows for a non-linear interaction between real photons. The physical process responsible for this is the existence of fluctuations - in the form of virtual particle-antiparticle pairs - within the QED vacuum. In the presence of strong fields, such as those generated in astrophysical contexts (e.g. ${ }^{28}$ ) or at next generation laser facilities $\left(\right.$ e.g. $\left.{ }^{29}\right)$, these virtual pairs are excited and can interact with probe photons passing through high field regions, which leads to a number of effects such as birefringence and photon splitting. For a more thorough overview of the subject we refer the reader to the reviews ${ }^{30-36}$ and references therein.

Born-Infeld electrodynamics was originally proposed as a theory to describe electrons with finite self energy, but its prominence in modern literature is due to the fact that the Born-Infeld action was rediscovered in the low-energy limit of string theory. ${ }^{27}$ Unlike Euler-Heisenberg, the Born-Infeld theory is the only known non-linear generalisation of electrodynamics which preserves many of the key results of linear Maxwellian theory, e.g. the lack of birefringence and shocks. ${ }^{37,38}$

The relevance of these theories in the present context is that under the presence of strong fields, the quantum vacuum obtains an effective refractive index (see for example ${ }^{31,39-41}$ ), and has an analogous effect on the propagation of a probe field to a polarizing medium. This gives rise to the possibility of performing "slow light" experiments, ${ }^{42,43}$ where non-linear electrodynamics effects can be measured via analysis of the velocity of light through regions of strong fields. More importantly for our purposes, using the set up of a probe field propagating through a strong background field, within non-linear electrodynamics, gives us an all optical set up with which to view the Abraham-Minkowski controversy. Since the "material" effects on the propagation of a probe field are entirely due to the background field, we remove any ambiguity or difficulty due to incorrect modelling of a real medium and have a completely characterisable system.

The rest of this paper is structured as follows. In section 2 we detail some of the key features of the Minkowski and Abraham energy-momentum tensors, and introduce the key notations which will be used throughout this work. In section 3 we obtain through variational principles the general form of energy-momentum tensors in non-linear electrodynamics, from which we can obtain the energy-momentum tensors of ordinary Maxwell electrodynamics in vacuum and a range of non-linear theories through appropriate choice of the Lagrangian density. In section 4 we take a closer look at the non-linear energy-momentum tensor and find some similarities between this and the Minkowski tensor, but also some key differences which highlight the effect non-linear theories have on the propagation of light in vacuum. In section 5 we discuss the implications of the results found in this paper, particularly the key result that within the approach taken, the Minkowski and Abraham forms are equivalent. This may suggest that there is no way to distinguish between the two in non-linear vacuum electrodynamics, but we indicate briefly how this could be achieved, before summarising.

The Einstein summation convention for repeated tensor indices is used throughout this paper (Greek indices run from $\mu=0, \ldots, 3)$, as are units in which $c=\epsilon_{0}=1$ unless explicitly stated otherwise.

\section{THE ABRAHAM AND MINKOWSKI ENERGY-MOMENTUM TENSORS}

The Abraham-Minkowski controversy is primarily discussed within the context of light-matter interactions, where we have a wave defined by the electomagnetic field tensor $F_{\mu \nu}=\partial_{\mu} A_{\nu}-\partial_{\nu} A_{\mu}$ (with gauge field $A_{\mu}$ ), which is incident on some material medium. The interaction between the wave and the medium is characterised by the electromagnetic excitation tensor,

$$
H^{\mu \nu}=\frac{1}{2} \chi^{\mu \nu \alpha \beta} F_{\alpha \beta},
$$


where $\chi^{\mu \nu \alpha \beta}$ encodes the electromagnetic properties of the medium. In general, $\chi^{\mu \nu \alpha \beta}$ may depend on the electromagnetic field tensor $F_{\mu \nu}$, giving rise to non-linear interactions between the field and itself within the medium, but this is distinct from the interactions which can occur between fields within the context of non-linear vacuum electrodynamics.

From the original derivations by Minkowski and Abraham, the key distinction between each approach essentially comes down to differences in the momentum density of the electromagnetic wave. Minkowski first proposed that this should be $\mathbf{g}_{M}=\mathbf{D} \times \mathbf{B}$, which would correspond to a total momentum of the wave of $p_{M}=n p$, where $p$ is the free-space momentum and $n$ is the refractive index of the medium. Written in a covariant manner as the energy-momentum tensor of the field this becomes (see for example ${ }^{44,45}$ ),

$$
\Theta^{\mu}{ }_{\nu}=H^{\mu \alpha} F_{\nu \alpha}-\frac{1}{4} \delta_{\nu}^{\mu} H^{\alpha \beta} F_{\alpha \beta} .
$$

The Minkowski form is not explicitly symmetric, which is typically thought to be a requirement of energymomentum tensors to ensure that angular momentum is conserved, and also is necessary within the context of coupling to gravity in general relativity. ${ }^{46}$ While it can be shown that non-symmetric energy-momentum tensors are not necessarily unphysical ( $\mathrm{see}^{45} \S \mathrm{II}$.C for a complete discussion of this point in the present context), it was this feature which led to a rival expression being introduced by Abraham, in which the momentum density is given (in the rest frame of the medium) by $\mathbf{g}_{A}=\mathbf{E} \times \mathbf{H} / c^{2}$ such that the total momentum of the wave is now $p_{A}=p / n$, and the Abraham energy-momentum tensor is,

$$
{\Omega^{\mu}}_{\nu}=\frac{1}{2}\left(F^{\mu \alpha} H_{\nu \alpha}+H^{\mu \alpha} F_{\nu \alpha}\right)-\frac{1}{4} \delta_{\nu}^{\mu} H^{\alpha \beta} F_{\alpha \beta}-\frac{1}{2}\left[u^{\mu} u_{\alpha}\left(F_{\nu \beta} H^{\alpha \beta}-H_{\nu \beta} F^{\alpha \beta}\right)+u_{\nu} u^{\alpha}\left(F^{\mu \beta} H_{\alpha \beta}-H^{\mu \beta} F_{\alpha \beta}\right)\right] .
$$

This necessitates the introduction of a time-like vector field $u^{\mu}\left(u^{2}=c^{2}\right)$ which corresponds to the velocity of the material medium. ${ }^{44}$

The standard procedures for obtaining (2) and (3) in the literature are generally quite distinct from one another, however a more unified approach for obtaining both forms was found in. ${ }^{47}$ These authors consider non-dispersive, linear media, where the constitutive relation (1) is written intrinsically using the language of forms as,

$$
H=Z(F),
$$

where $H=\frac{1}{2} H_{\mu \nu} d x^{\mu} \wedge d x^{\nu}$ and $F=\frac{1}{2} F_{\mu \nu} d x^{\mu} \wedge d x^{\nu}$ are the 2-forms, or tensor fields, with components $H_{\mu \nu}$ and $F_{\mu \nu}$ respectively, i.e. the excitation and electromagnetic field "tensors". They find that obtaining one or the other of the Abraham or Minkowski forms of the energy-momentum tensor is entirely dependent on whether or not the constitutive tensor field $Z$ depends on the metric (tensor field) $g$. When $Z$ has explicit dependence on $g$ the Abraham tensor appears naturally from variational principles, and when it is independent of $g$ the (symmetrised) Minkowski form is obtained.

\subsection{Trace and divergence}

It is useful here to discuss some of the features both of the energy-momentum tensors share, and where they differ. By inspection of (2) and (3) it is easy to see that they are both traceless,

$$
\Theta_{\mu}^{\mu}=0=\Omega_{\mu}^{\mu}
$$

which is simply a statement of the massless nature of photons, and that deviations of the speed of the wave from the speed of light $c$ are due to the influence of the material medium on the wave.

Taking the divergence of the Minkowski tensor (2) we obtain the momentum-balance equation, ${ }^{45}$

$$
\partial_{\mu} \Theta^{\mu}{ }_{\nu}+\mathcal{F}_{\nu}^{(m)}+\mathcal{F}_{\nu}^{(J)}=0
$$

where $\mathcal{F}^{(m)}{ }_{\nu}$ and $\mathcal{F}^{(J)}{ }_{\nu}$ are the effective 4-force densities due to, respectively, the macroscopic transfer of energy from the field to the medium, and from external currents and charges. ${ }^{45}$ Under the assumption of a homogeneous, 
time-independent medium and a closed system such that $\mathcal{F}^{(m)}{ }_{\nu}=\mathcal{F}^{(J)}{ }_{\nu}=0$, we see that the Minkowski tensor, though not in general symmetric, still conserves 4-momentum.

For the divergence of the Abraham tensor, it is first useful to write (3) in terms of (2),

$$
{\Omega^{\mu}}_{\nu}=\Theta^{\mu}{ }_{\nu}+\frac{1}{2}\left(F^{\mu \alpha} H_{\nu \alpha}-H^{\mu \alpha} F_{\nu \alpha}\right)-\frac{1}{2 c^{2}}\left[u^{\mu} u_{\alpha}\left(F_{\nu \beta} H^{\alpha \beta}-H_{\nu \beta} F^{\alpha \beta}\right)+u_{\nu} u^{\alpha}\left(F^{\mu \beta} H_{\alpha \beta}-H^{\mu \beta} F_{\alpha \beta}\right)\right],
$$

such that we have, ${ }^{45}$

$$
\partial_{\mu} \Omega^{\mu}{ }_{\nu}+\mathcal{F}^{(A)}{ }_{\nu}=\partial_{\mu} \Theta^{\mu}{ }_{\nu} .
$$

The appearance on the left hand side of the so-called Abraham 4 -force density, $\mathcal{F}^{(A)}{ }_{\nu}$, gives the Minkowski and Abraham forms a key difference. Even under circumstances where $\partial_{\mu} \Theta^{\mu}{ }_{\nu}=0$, the 4-momentum is generally not conserved in Abraham's approach, i.e. $\partial_{\mu} \Omega^{\mu}{ }_{\nu} \neq 0$, due to the fact that the 4 -force density is non-zero even with extremely simple configurations of waves and materials. Since, then, the Minkowski tensor has a preferential status due to the fact that it can be conserved purely via the symmetry conditions of the medium, it has been adopted by some authors as the more convenient form of the energy-momentum tensor. ${ }^{18,45}$ It is reasonable then to ask whether or not this status will be retained by the Minkowski tensor, and how we can interpret Abraham, when viewing the debate from the perspective of non-linear vacuum electrodynamics.

\section{EXACT ENERGY-MOMENTUM TENSOR}

Theories of non-linear vacuum electrodynamics give us the opportunity to approach the Abraham-Minkowski debate from an entirely new perspective, using an all-optical set up. In such theories the vacuum acts as a polarizable medium when strong electromagnetic fields are present. In the usual context of light-matter interactions, the presence of the medium breaks the Lorentz invariance of the system. However, this is not the case for non-linear vacuum electrodynamics, where Lorentz invariance is strictly preserved. We can therefore obtain a general description of such theories by defining the Lagrangian density, $\mathcal{L}$, in terms of Lorentz invariant contractions of the electromagnetic field tensor $F_{\mu \nu}$ and the dual tensor $\widetilde{F}^{\mu \nu}=1 / 2 \epsilon^{\mu \nu \alpha \beta} F_{\alpha \beta}$ (where $\epsilon^{\mu \nu \alpha \beta}$ is the Levi-Civita symbol). The only invariants which can be built from these are,

$$
X=-\frac{1}{4} F^{\mu \nu} F_{\mu \nu}=\frac{1}{2}\left(\frac{|\mathbf{E}|^{2}}{c^{2}}-|\mathbf{B}|^{2}\right), \quad Y=-\frac{1}{4} \tilde{F}^{\mu \nu} F_{\mu \nu}=\frac{\mathbf{E} . \mathbf{B}}{c},
$$

such that for non-linear vacuum electrodynamics, $\mathcal{L}=\mathcal{L}(X, Y)$.

Field equations and conservation laws are obtained through variation of the classical action,

$$
S=\int d^{4} z \sqrt{-G} \mathcal{L}(X, Y)
$$

where $\sqrt{-G} d^{4} z$ is the spacetime 4 -volume, and $G=\operatorname{det} g_{\mu \nu}$ is the determinant of the metric tensor which we define with mostly negative signature, $g^{\mu \nu}=\operatorname{diag}(1,-1,-1,-1)$. We have explicitly assumed a closed system, with no external free charges.

With this notation the Lagrangian densities of Maxwell, Born-Infeld, and (lowest order) Euler-Heisenberg theories are, respectively,

$$
\begin{aligned}
\mathcal{L}_{\text {Max }}(X, Y) & =X \\
\mathcal{L}_{B I}(X, Y) & =\frac{1}{\kappa^{2}}\left(1-\sqrt{1-2 \kappa^{2} X-\frac{\kappa^{2}}{4} Y^{2}}\right), \\
\mathcal{L}_{E H}(X, Y) & =X+\frac{2 \alpha^{2}}{45 m^{4}}\left(4 X^{2}+7 Y^{2}\right)
\end{aligned}
$$

where $\alpha \simeq 1 / 137$ is the fine structure constant, $m$ is the electron mass and $\kappa$ is an unknown constant. 
By varying the action (10) with respect to the gauge field $A^{\mu}$ we arrive at the equation of motion,

$$
\partial_{\mu} H^{\mu \nu}=0,
$$

where the electromagnetic excitation tensor is defined as,

$$
H^{\mu \nu} \equiv \frac{\partial \mathcal{L}}{\partial X} F^{\mu \nu}+\frac{\partial \mathcal{L}}{\partial Y} \widetilde{F}^{\mu \nu}
$$

We also have, for the electromagnetic field tensor, $F^{\mu \nu}$, the usual Bianchi identity,

$$
\partial_{\mu} F_{\nu \lambda}+\partial_{\lambda} F_{\mu \nu}+\partial_{\nu} F_{\lambda \mu}=0
$$

In linear Maxwellian electrodynamics where the Lagrangian density is (11), the excitation tensor (15) reduces to $H^{\mu \nu} \stackrel{\text { Maxwell }}{=} F^{\mu \nu}$, and in general $H^{\mu \nu}$ encodes the non-linear interactions between the electromagnetic fields. In the standard view of the Abraham-Minkowski debate, $H^{\mu \nu}$ incorporates the macroscopic properties of the material medium through which an electromagnetic field is propagating, such as the magnetisation and polarisation. In the all optical set up it plays an analogous role in that it describes the influence of a non-linear electromagnetic theory on the propagation of light through vacuum.

The energy-momentum tensor, $T^{\mu \nu}$, is found through varying the action (10) with respect to the metric tensor, $g^{\mu \nu}$, such that,

$$
\delta S=\frac{1}{2} \int d^{4} x \sqrt{-G} T^{\mu \nu} \delta g_{\mu \nu},
$$

which gives,

$$
T^{\mu}{ }_{\nu}=H^{\mu \alpha} F_{\nu \alpha}+\delta_{\nu}^{\mu} \mathcal{L} .
$$

The energy-momentum tensor obtained here is exact, and applies to all non-linear extensions to Maxwell theory. There is already an obvious resemblance to the form of the Minkowski tensor (2) (though it should be noted that (18) is symmetric, as follows from (15)). However, only in the special case where $\mathcal{L}=-\frac{1}{4} H^{\alpha \beta} F_{\alpha \beta}$ do we have that

$$
\mathcal{L}=-\frac{1}{4} H^{\alpha \beta} F_{\alpha \beta}: \quad T^{\mu}{ }_{\nu}=\Theta^{\mu}{ }_{\nu} .
$$

This particular choice of Lagrangian density holds for conformally invariant theories, and is equivalent to ordinary linear Maxwellian electrodynamics in a medium. For a general medium, where $H^{\mu \nu}$ is not defined by (15), this would lead to a breaking of the Lorentz invariance. So, without this particular choice of Lagrangian, there will be a deviation from the results of Minkowski, though this will not affect the momentum density.

\section{PROPERTIES OF THE NON-LINEAR ENERGY-MOMENTUM TENSOR}

As for the Minkowski and Abraham forms, we now look at some of the key properties of the non-linear energymomentum tensor. Firstly, it is clear that in general (18) is not trace free,

$$
T_{\mu}^{\mu}=H^{\mu \alpha} F_{\mu \alpha}+4 \mathcal{L} .
$$

The tracelessness of the energy-momentum tensor is a direct consequence of the massless nature of photons. In ordinary Maxwell electrodynamics in medium, any change in the momentum of the field is as a result of the influence of the material on propagation. In non-linear electrodynamics, where there is no direct medium, only an excited vacuum state, changes in momentum can be thought of as being due to photons within the electromagnetic field acquiring an effective mass.

Secondly, taking the divergence of (18) we have, using (14),

$$
\partial_{\mu} T_{\nu}^{\mu}=H^{\mu \alpha} \partial_{\mu} F_{\nu \alpha}+\partial_{\nu} \mathcal{L}
$$


Expanding the last term, and using the definition (15),

$$
\partial_{\nu} \mathcal{L}=-\frac{1}{2} H^{\alpha \beta} \partial_{\nu} F_{\alpha \beta},
$$

which, along with the Bianchi identity (16) can be used to show that the energy-momentum tensor of non-linear electrodynamics is fully conserved,

$$
\partial_{\mu} T^{\mu}{ }_{\nu}=0 .
$$

This has an obvious qualitative similarity to the divergence free nature of the Minkowski tensor (2) when we consider a closed system with a homogeneous and time-independent medium. This is, in part, due to the fact that for the non-linear energy-momentum tensor, we are essentially looking at a completely closed system, as the effective medium through which the applied field $F^{\mu \nu}$ is propagating is completely accounted for by the excitation tensor (15). However, unlike in the case of Minkowski, no further assumptions about the symmetries of the background fields, and as a consequence the vacuum which plays the role of the medium, have been made the energy-momentum tensor is conserved in general in the absence of free charges. This highlights the distinct advantage of approaching this problem from the perspective of non-linear electrodynamics. The entire system is described by fields, which we can fully characterise. Contrasting this with the results of the Abraham tensor in a medium - where even for closed systems with simple media there remains a non-zero divergence term in (8), given by the Abraham 4-force $\mathcal{F}_{\mu}^{(A)}{ }_{\mu}$ - it would appear that the latter has no obvious role in non-linear vacuum electrodynamics.

\section{DISCUSSION}

From our analysis above, there appears to be a much more natural interpretation of the Minkowski tensor in the present context. However, it can be shown that the definition of the energy-momentum tensor (18) conceals within it a unification of the Minkowski and Abraham forms in the context of non-linear vacuum electrodynamics. By using the excitation tensor (15) in the Abraham tensor (3) or (7) we find that all additional terms drop out and we are left with ${\Omega^{\mu}}_{\nu}=\Theta^{\mu}{ }_{\nu}$. This would appear to indicate that we cannot distinguish between the Abraham and Minkowski forms of the energy-momentum tensor using the approach taken in this paper.

However, there are some distinctions which must be made between the non-linear energy-momentum tensor (18) and those of Minkowski (2) and Abraham (3). Following the arguments of various authors (e.g. ${ }^{16,18,48,49}$ ), the differences between Abraham and Minkowski are typically attributed to how we split the total energymomentum tensor into electromagnetic and material components, and that in fact,

$$
\Theta^{\mu}{ }_{\nu}+T_{M, m a t}{ }_{\nu}=\Omega^{\mu}{ }_{\nu}+T_{A, m a t}{ }_{\nu}{ }_{\nu} .
$$

For our general non-linear electromagnetic tensor (18), we are dealing purely with fields. This means that the electromagnetic contribution to the full energy-momentum tensor is the only component. The equivalence of the Minkowski and Abraham tensors in non-linear electrodynamics with the definition of the excitation tensor (15) could then simply be a statement of the fact that the energy-momentum tensor (18) can be interpreted as the full energy-momentum tensor, giving some further credibility to (24). This could also indicate that there may still be room to distinguish between the Abraham and Minkowski tensors in non-linear vacuum electrodynamics - by separating (18) into terms describing explicitly the "medium", or the background fields in the vacuum, and the propagating wave. In this way we may be able to isolate "wave-only" terms, which would be more directly comparable to the Minkowski and Abraham tensors. We will visit this subject elsewhere. ${ }^{50}$

In summary, the debate surrounding the momentum of light in a material medium has led to many claims and counter claims being made, with a possible resolution lying in the consistent partition of the full energymomentum tensor into electromagnetic and material contributions. We have approached this problem from the perspective of non-linear electrodynamics, where the role of the medium is played by the vacuum excited in the presence of strong background fields. We have shown that for a general non-linear theory of electrodynamics, there is a clear deviation away from the results of both Abraham and Minkowski, but that distinguishing between the two tensors will require a more detailed analysis. ${ }^{50}$ 


\section{ACKNOWLEDGMENTS}

The authors would like to thank the organisers at SPIE for hosting the Optics and Optoelectronics 2017 event, and for the invitation to present this work. AJM would like to thank EPSRC and University of Strathclyde for a DTP studentship. This work was supported by the UK EPSRC (grant no. EP/N028694/1) and by EC through Laserlab-Europe, H2020 EC-GA 654148 and EC H2020 EuPRAXIA (grant no.653782).

\section{REFERENCES}

[1] Minkowski, H., "Die grundlagen für die elektromagnetischen vorgënge in bewegten körpern," Nachr. $d$. König. Gesellsch. d. Wiss. zu Gttingen, Math-phys. Klasse 53, 1978-1979 (1908).

[2] Minkowski, H., "Die grundgleichungen für die elektromagnetischen vorgänge in bewegten körpern," Math. Ann. 68(4), 472-525 (1910).

[3] Abraham, M., "Zur elektrodynamik bewegter körper [on the electrodynamics of moving bodies]," Rend. Circ. Mat. Palermo 28(1), 1-28 (1909).

[4] Abraham, M., "Sullelettrodinamica di minkowski [on minkowskis electrodynamics]," Rend. Circ. Mat. Palermo 30(1), 33-46 (1910).

[5] Barnett, S. M. and Loudon, R., "The enigma of optical momentum in a medium," Philos. Trans. R. Soc. London, Ser. A 368(1914), 927-939 (2010).

[6] Laue, M. V., "Zur minkowskischen elektrodynamik der bewegten körper," Z. Phys. 128(3), 387-394 (1950).

[7] Brevik, I., "Experiments in phenomenological electrodynamics and the electromagnetic energy-momentum tensor," Phys. Rep. 52(3), 133-201 (1979).

[8] Campbell, G. K., Leanhardt, A. E., Mun, J., Boyd, M., Streed, E. W., Ketterle, W., and Pritchard, D. E., "Photon recoil momentum in dispersive media," Phys. Rev. Lett. 94(17), 170403 (2005).

[9] Padgett, M. J., "On diffraction within a dielectric medium as an example of the minkowski formulation of optical momentum," Optics Express 16(25), 20864-20868 (2008).

[10] Mansuripur, M., "Comment on observation of a push force on the end face of a nanometer silica filament exerted by outgoing light," Phys. Rev. Lett. 103(1), 019301 (2009).

[11] James, R. P., "A simplest case experiment resolving abraham-minkowski controversy on electromagnetic momentum in matter," Proc. Natl. Acad. Sci. U.S.A. 61(3), 1149 (1968).

[12] Walker, G. B., Lahoz, D. G., and Walker, G., "Measurement of the abraham force in a barium titanate specimen," Can. J. Phys. 53(23), 2577-2586 (1975).

[13] Skobel'tsyn, D. V., "The momentum-energy tensor of the electromagnetic field," Sov. Phys. Usp. 16(3), 381 (1973).

[14] Lai, H. M., "Electromagnetic momentum in static fields and the abraham-minkowski controversy," Am. J. Phys. 48(8), 658-659 (1980).

[15] She, W., Yu, J., and Feng, R., "Observation of a push force on the end face of a nanometer silica filament exerted by outgoing light," Phys. Rev. Lett. 101(24), 243601 (2008).

[16] Penfield, P. and Haus, H. A., [Electrodynamics of moving media.], MIT, Cambridge, MA (1967).

[17] Robinson, F. N. H., "Electromagnetic stress and momentum in matter," Phys. Rep. 16(6), 313-354 (1975).

[18] Barnett, S. M., "Resolution of the abraham-minkowski dilemma," Phys. Rev. Lett. 104(7), 070401 (2010).

[19] Pfeifer, R. N., Nieminen, T. A., Heckenberg, N. R., and Rubinsztein-Dunlop, H., "Colloquium: Momentum of an electromagnetic wave in dielectric media," Rev. of Mod. Phys. 79(4), 1197 (2007).

[20] Balazs, N. L., "The energy-momentum tensor of the electromagnetic field inside matter," Phys. Rev. 91(2), 408 (1953).

[21] Arnaud, J. A., "Radiation force on multilayer media," Opt. Commun. 7(4), 313-316 (1973).

[22] Arnaud, J. A., "Momentum of photons," Am. J. Phys. 42(1), 71-73 (1974).

[23] Heisenberg, W. and Euler, H., "Folgerungen aus der diracschen theorie des positrons," Z. Phys. 98(11-12), 714-732 (1936).

[24] Heisenberg, W. and Euler, H., "Consequences of dirac theory of the positron," arXiv preprint physics/0605038 (2006).

[25] Schwinger, J., "On gauge invariance and vacuum polarization," Phys. Rev. 82(5), 664 (1951). 
[26] Born, M. and Infeld, L., "Foundations of the new field theory," Proc. R. Soc. London, Ser. A 144(852), 425-451 (1934).

[27] Fradkin, E. and Tseytlin, A. A., "Non-linear electrodynamics from quantized strings," Phys. Lett. B 163(14), 123-130 (1985).

[28] Lai, D. and Ho, W. C., "Polarized x-ray emission from magnetized neutron stars: signature of strong-field vacuum polarization," Phys. Rev. Lett. 91(7), 071101 (2003).

[29] "Extreme light infrastructure nuclear physics." http://www.eli-np.ro/ (2017).

[30] Dunne, G. V., "Extreme quantum field theory and particle physics with izest," Eur. Phys. J. Special Topics 223(6), 1055-1061 (2014).

[31] Dittrich, W. and Gies, H., [Probing the quantum vacuum: perturbative effective action approach in quantum electrodynamics and its application], vol. 166, Springer Science \& Business Media (2000).

[32] Marklund, M., "Fundamental optical physics: Probing the quantum vacuum," Nature Photonics 4(2), 72 (2010).

[33] Homma, K., Habs, D., Mourou, G., Ruhl, H., and Tajima, T., "Opportunities of fundamental physics with high-intensity laser fields," Prog. Theor. Phys. Supp. 193, 224-229 (2012).

[34] Heinzl, T., Liesfeld, B., Amthor, K.-U., Schwoerer, H., Sauerbrey, R., and Wipf, A., "On the observation of vacuum birefringence," Opt. Commun. 267(2), 318-321 (2006).

[35] Gies, H., "Strong laser fields as a probe for fundamental physics," Eur. Phys. J. D 55(2), 311-317 (2009).

[36] Di Piazza, A., Müller, C., Hatsagortsyan, K., and Keitel, C., "Extremely high-intensity laser interactions with fundamental quantum systems," Rev. Mod. Phys. 84(3), 1177 (2012).

[37] Boillat, G., "Nonlinear electrodynamics: Lagrangians and equations of motion," J. Math. Phys. 11(3), 941-951 (1970).

[38] Gibbons, G. and Herdeiro, C., "Born-infeld theory and stringy causality," Phys. Rev. D 63(6), 064006 (2001).

[39] Dittrich, W. and Gies, H., "Light propagation in nontrivial qed vacua," Phys. Rev. D 58(2), 025004 (1998).

[40] Dinu, V., Heinzl, T., Ilderton, A., Marklund, M., and Torgrimsson, G., "Vacuum refractive indices and helicity flip in strong-field qed," Phys. Rev. D 89(12), 125003 (2014).

[41] Affleck, I., "Photon propagation in a plane-wave field," J. Phys. A 21(3), 693 (1988).

[42] Flood, S. P. and Burton, D. A., "Testing vacuum electrodynamics using slow light experiments," Europhys. Lett. 100(6), 60005 (2013).

[43] Flood, S. and Burton, D., "Strong fields and slow light," Central Laser Facility (2015).

[44] Obukhov, Y. N., "Electromagnetic energy and momentum in moving media," Ann. Phys. 17(9-10), 830-851 (2008).

[45] Ramos, T., Rubilar, G. F., and Obukhov, Y. N., "First principles approach to the abraham-minkowski controversy for the momentum of light in general linear non-dispersive media," J. Optics 17(2), 025611 (2015).

[46] Wald, R. M., [General relativity], University of Chicago press, Chicago, IL (2010).

[47] Dereli, T., Gratus, J., and Tucker, R. W., "New perspectives on the relevance of gravitation for the covariant description of electromagnetically polarizable media," J. Phys. A 40(21), 5695 (2007).

[48] Peierls, R., "The momentum of light in a refracting medium," in [Proc. R. Soc. London A], 347(1651), 475-491 (1976).

[49] Israel, W., "Relativistic effects in dielectrics: an experimental decision between abraham and minkowski?," Phys. Lett. B 67(1), 125-128 (1977).

[50] Macleod, A. J. and Noble, A., "In preparation," . (2017). 